\title{
EDITORIAL
}

\section{Dexmedetomidine: Its fascination, fad, and facts in neuroanaesthesia practice!}

Dexmedetomidine is a recently introduced drug in India, which has gained immense popularity among the anaesthesiologists. The sedation and analgesic activity without producing respiratory depression has been considered as the greatest advantage of the drug when compared with benzodiazepines and opioids, and this property is also responsible for its popularity. It has been originally introduced in the United States and approved for use in the intensive care as a sedative agent in the critically ill patients requiring mechanical ventilation to be administered as intravenous infusion by the Food and Drug Administration in 1998. ${ }^{[1]}$ Dexmedetomidine has found its ways from intensive care unit to various places including the operation rooms, catheterization laboratories, radiological suite, etc., and its route of administration also widened from the intravenous route to inclusion in regional blocks as adjuvants, as an oral premedication agent, etc. Dexmedetomidine use has also expanded to various surgical specialties including cardiac surgical, pediatric surgical, regional anaesthesia, and neurosurgical practice. Its use has been found to reduce the needs for opioids and anaesthetics intraoperatively.

It has a potent sedative, anxiolytic, and analgesic activity, and it is a selective $\alpha-2$ agonist agent. $\alpha-2$ receptors are present in large concentrations in the arousal areas of the brain as well as vascular smooth vessels. In the central nervous system (CNS), activation of $\alpha-2$ receptors reduces norepinephrine release, reduced sympathetic activity and sedation. In the cortical blood vessels activation of presynaptic $\alpha$-2-adrenoreceptors decrease norepinephrine release, whereas postsynaptic $\alpha-2-$ adrenoreceptors may directly increase vascular smooth muscle tone. Hence, infusions of dexmedetomidine may have both direct (i.e. $\alpha-2$ agonist-mediated increases in calcium flux, triggering vascular smooth muscle constriction) and $\alpha-2$ indirect (changes in central sympathetic activity and decreased cerebral metabolic rate) effects on cerebral blood flow (CBF). ${ }^{[2]}$

\begin{tabular}{|l|l|}
\hline \multicolumn{2}{|c|}{ Access this article online } \\
\hline Quick Response Code: & Website: \\
\hline & www.jnaccjournal.org \\
\cline { 2 - 2 } & \\
\hline
\end{tabular}

In the peripheral blood vessels, effects of dexmedetomidine depends on the balance between central and peripheral mechanisms. By central mechanisms, it reduces sympathetic outflow and causes hypotension, whereas peripheral direct action of vasoconstriction may lead to hypertension. Thus, loading dose of dexmedetomidine usually cause systemic hypertension, followed by hypotension. The degree of haemodynamic effects depends on the dose and fluid status of the patient.

Though the drug has been used in the critical care setting in the medical patients, its use in neuroanaesthesia practice is limited. Hence, it is imperative to understand the CNS effects of dexmedetomidine in neurological patients. The basic principles of neuroanaesthesia are prevent cerebral ischaemia, control of increased intracranial pressure (ICP), haemodynamic stability during surgery and rapid awakening at the end of surgery. ${ }^{[3]}$ In addition intraoperative neuroprotection is also a necessity. Prevention of cerebral ischaemia is achieved by optimizing the cerebral perfusion pressure, cerebral oxygenation and control of intraoperative ICP is achieved by preventing cerebral vasodilation, cerebral oedema, and brain swelling. Modern neurosurgery lays lot of emphasis on the functional preservation and minimally invasive surgery. The goals in managing these patients addition to the above basic necessities are awakening of patients during surgery, monitoring of electrocorticogram (ECoG), intraoperative evoked potential monitoring, etc. Hence, an anaesthetic agent must be satisfying the above needs of the neuroanaesthetist and should not be detrimental to the outcome.

Infusion of dexmedetomidine was found not to increase the ICPs measured via lumbar catheter in spinal subarachnoid space following pituitary surgery. All these patients had normal cerebrospinal fluid pressure. ${ }^{[4]}$ However, its effects on patients with raised ICP have not been evaluated well. In a small series of 12 patients with head injury, dexmedetomidine infusion did not result in increased ICP. However, all of them had ICP of $<20 \mathrm{mmHg} .{ }^{[5]}$ It needs to be seen that the drug is safe in patients with moderate or severely raised ICP.

The effects of dexmedetomidine on the CBF have been well established in many studies. Both the low and high dose of the drug has been found to reduce the global as 
well as regional CBF in cortical and subcortical areas. ${ }^{[6]}$ This reduction in CBF cannot be explained by the modest reduction in heart rate and blood pressure the drug causes by acting on the systemic vasculature. This reduction was thought to be direct cerebral vasoconstriction mediated by $\alpha-2$ receptor activation. The reduction can be considered harmful in patients who depend on the CBF like acute stroke as well as cerebral vasospasm whereas it can be beneficial in states like vasogenic cerebral oedema. Dexmedetomidine sedation has been found to reduce cerebral metabolic rate of oxygen. This can also be a contributing factor for reduction in CBF. Though the action of the drug causes concern of cerebral ischaemia in neurosurgical patients, a small study in acute brain injury has shown that lactate/pyruvate ratio was comparable with propofol infusion well maintained favoring its use. ${ }^{[7]}$ There are certain areas of cerebral haemodynamic where limited knowledge is available with dexmedetomidine use. The most important of these is carbon-dioxide $\left(\mathrm{CO}_{2}\right)$ reactivity and cerebral autoregulation. Even though the $\mathrm{CO}_{2}$ reactivity is maintained in volunteers, the effects on impaired auto regulation need to be determined. There is limited data on this aspect.

Haemodynamic stability is one of the important goals of neuroanaesthesia. Intraoperative use of dexmedetomidine infusion during a neurosurgical procedure has been found to maintain the haemodynamics better despite the feared hypotension, reduces the requirement of opioids and anaesthetic agents. ${ }^{[8]}$ Our experience also showed dexmedetomidine infusion prevents haemodynamic response to skull pin insertions as well as reduced requirement of opioids and anti hypertensives during transphenoidal pituitary resection surgery. There are two major disadvantages of dexmedetomidine; hypotension and severe bradycardia and can induce sinus arrest. ${ }^{[9]}$ In patients with raised ICP with bradycardia as well as patients on b-blockers, administration of dexmedetomidine can precipitate sinus arrest. Hypotension is usually caused by the loading dose of the drug especially in hypovolemic patients. Neurosurgical patients can be dehydrated in the preoperative period especially those on diuretics. Hypotension can be avoided by giving only the maintenance dose of the drug, skipping the loading dose and adequately hydrating the patient.

The greatest advantage of dexmedetomidine is its conscious sedation with rapid recovery with analgesic action and ability to test neurological intactness in patients undergoing neurosurgery. Dexmedetomidine use is widespread during functional neurosurgery like deep brain stimulation as it maintains the abnormal movements, neuronavigational procedures and awake craniotomy for tumor and epilepsy surgery. ${ }^{[10]}$ It has also been found to be useful in coiling of aneurysms in neuroradiological suite. ${ }^{[11]}$ There are limited randomized data available in comparison with propofol/remifentanyl combination. Dexmedetomidine has been found effective for sedation in both adults and pediatric patients undergoing magnetic resonant imaging. ${ }^{[12]}$

Intraoperative ECoG and evoked potential monitoring are few specialized procedures that are usually affected by the administration of anaesthetic drugs. Dexmedetomidine infusion did not affect the quality of ECoG as it produces electroencephalography like normal sleep pattern. ${ }^{[12]}$ Moreover, experience during scoliosis surgery has shown that dexmedetomidine preserved the somatosensory and motor evoked potentials. ${ }^{[13]}$

More often than not neuroanaesthesiologist encounters difficult intubations especially in diseases of the cervical spine such as cervical trauma and atlantoaxial dislocation. Awake fiberoptic intubation as well as awake positioning may be required in these cases. Dexmedetomidine infusion during the procedure has been found to be safe with better patient comfort, oxygen saturation without the fear of loss of airway. ${ }^{[14]}$

As with many intravenous and inhalational anaesthetic drugs, dexmedetomine has also been found to have neuroprotective properties in animal studies. It helps in preventing schema reperfusion injury, apoptosis. ${ }^{[15]}$ The clinical utility of neuroprotection has not been studied so far.

Patients in neurocritical care are different from other intensive care in that they may require good sedation for control of the raised ICP. In addition, they need to be monitored neurologically with pupillary changes as well as neurological testing. High dose opioids with benzodiazepines are usually the practice in many neurointensive care units. They can cause haemodynamic instability, interferes with the pupillary assessment and prolonged use may cause withdrawal features. Dexmedetomidine can counter the effects of high dose of these drugs. It helps in neurological assessment and does not interfere in the pupillary assessment. It is helpful in weaning the patients who are at risk of opioid withdrawal syndrome. ${ }^{[16]}$ The duration of infusion of dexmedetomidine is not clearly defined. Even though, it is recommended for short term $(<24 \mathrm{~h})$, reports of prolonged infusion without adverse event exists in the literature.

In conclusion, dexmedetomidine is a very useful drug, especially in functional neurosurgery, awake craniotomies, sedation for radiological procedures and short term use in neurocritical care. However, it should be borne in mind its haemodynamic adverse effects like hypotension and bradycardia when choosing this drug for neurologically ill. At present, there are limited studies related to the use of dexmedetomidine in 
Neuroanaesthesia and neurocritical care and many of them are case series. There are no definitive guideline that exist favoring any strong recommendation for the use this drug in Neuroanaesthesia practice.

\section{Sethuraman Manikandan}

Department of Anaesthesiology, Sree Chitra Tirunal Institute for Medical Sciences and Technology, Trivandrum, Kerala, India

Address for correspondence:

Dr. Sethuraman Manikandan,

A-4, NFQ, SCTIMST, Poonthi Road, Kumarapuram,

Trivandrum - 695 011, Kerala, India.

E-mail: kanmanisethu@gmail.com

\section{REFERENCES}

1. Precedex (dexmedetomidine) Package Insert. Lake Forest, IL: Hospira Inc.; 2008.

2. Bekker A, Sturaitis MK. Dexmedetomidine for neurological surgery. Neurosurgery 2005;57:1-10.

3. Dinsmore J. Anaesthesia for elective neurosurgery. $\mathrm{Br} \mathrm{J}$ Anaesth 2007;99:68-74.

4. Talke P, Tong C, Lee HW, Caldwell J, Eisenach JC, Richardson CA. Effect of dexmedetomidine on lumbar cerebrospinal fluid pressure in humans. Anesth Analg 1997;85:358-64.

5. Grille P, Biestro A, FariFa G, Miraballes R. Effects of dexmedetomidine on intracranial haemodynamics in severe head injured patients. Neurocirugia (Astur) 2005;16:411-8.

6. Prielipp RC, Wall MH, Tobin JR, Groban L, Cannon MA, Fahey FH, et al. Dexmedetomidine-induced sedation in volunteers decreases regional and global cerebral blood flow. Anesth Analg 2002;95:1052-9.

7. James ML, Olson DM, Graffagnino C. A pilot study of cerebral and haemodynamic physiological changes during sedation with dexmedetomidine or propofol in patients with acute brain injury. Anaesth Intensive Care 2012;40:949-57.

8. Soliman RN, Hassan AR, Rashwan AM, Omar AM. Prospective, randomized study to assess the role of dexmedetomidine in patients with supratentorial tumors undergoing craniotomy under general anaesthesia. Middle East J Anaesthesiol 2011;21:325-34

9. Bindra TK, Sarin SS, Gupta R, Shubhdeep. Sinus arrest with intrathecal dexmedetomidine. Indian J Anaesth 2014;58:227-8.

10. Ard JL Jr, Bekker AY, Doyle WK. Dexmedetomidine in awake craniotomy: A technical note. Surg Neurol 2005;63:114-6.

11. Lee $\mathrm{HH}$, Jung $\mathrm{YJ}$, Choi BY, Chang $\mathrm{CH}$. Usefulness of Dexmedetomidine during Intracerebral Aneurysm Coiling. J Korean Neurosurg Soc 2014;55:185-9.

12. Mason KP, Lerman J. Review article: Dexmedetomidine in children: Current knowledge and future applications. Anesth Analg 2011;113:1129-42.

13. Bala E, Sessler DI, Nair DR, McLain R, Dalton JE, Farag E. Motor and somatosensory evoked potentials are well maintained in patients given dexmedetomidine during spine surgery. Anaesthesiology 2008;109:417-25.

14. He XY, Cao JP, He Q, Shi XY. Dexmedetomidine for the management of awake fibreoptic intubation. Cochrane Database Syst Rev 2014;1:CD009798.

15. Kakinohana M, Oshiro M, Saikawa S, Nakamura S, Higa T, Davison $\mathrm{KJ}$, et al. Intravenous infusion of dexmedetomidine can prevent the degeneration of spinal ventral neurons induced by intrathecal morphine after a noninjurious interval of spinal cord ischaemia in rats. Anesth Analg 2007;105:1086-93.

16. Grof TM, Bledsoe KA. Evaluating the use of dexmedetomidine in neurocritical care patients. Neurocrit Care 2010;12:356-61.

How to cite this article: Manikandan S. Dexmedetomidine: Its fascination, fad, and facts in neuroanaesthesia practice!. J Neuroanaesthesiol Crit Care 2014;1:163-5.

Source of Support: Nil, Conflict of Interest: None declared. 